\title{
A Single-Image Super-Resolution Method for Texture Interpolation
}

\author{
Yaron Kalit and Moshe Porat
}

\begin{abstract}
In recent years, a number of super-resolution techniques have been proposed. Most of these techniques construct a high resolution image by either combining several low resolution images at sub-pixel misalignments or by learning correspondences between low and high resolution image pairs. In this paper we present a stochastic super-resolution method for color textures from a single image. The proposed algorithm takes advantage of the repetitive nature of textures and the existence of several similar patches within the texture, as well as the color-intensity correlation that often exist in natural images. In the first step of the algorithm the intensity component is interpolated. For each pixel, the missing value is chosen according to a probability distribution constructed from a measure of similarity to other patches in the texture as well as from local features and patch color similarity. In the second stage, the color components are interpolated in a similar manner, using patches of the color channels as well as the already interpolated intensity values. Our conclusion is that the proposed approach outperforms presently available methods.
\end{abstract}

Index Terms-Image processing, super resolution, texture interpolation, color zooming.

\section{INTRODUCTION}

Interpolation is one of the fundamental tasks in image processing. Its applications range from medical and astronomical image processing to magnification of details in images acquired from surveillance cameras. The popular interpolation methods such as the bi-linear, bi-cubic and nearest neighbor methods are convolution based, space invariant methods. They are widely used due to their low computational complexity and simple implementation. Since these algorithms function in essence as low-pass filters, they tend to introduce blurring and blocking artifacts, which are visible especially in areas containing high frequency content such as edges as well as in textures. Several classes of more sophisticated algorithms were proposed in recent years [1]-[3]. Most of them adapt to the image and attempt to reconstruct the high frequency content in a manner that preserves the edges and introduces as few artifacts as possible.

An important class of algorithms known as super-resolution (SR) uses multiple low resolution images in order to reconstruct a high resolution image [4]. The classical SR techniques combine several low resolution images at sub-pixel misalignments. Different kinds of SR algorithms

Manuscript received June 15, 2012; revised July 12, 2012.

This research was supported in part by Technion's fund \#7110134 and by the Ollendorff Minerva Center. Minerva is funded through the BMBF.

The authors are with the Department of Electrical Engineering, Technion, Haifa 32000, Israel (e-mail: ykalit@tx.technion.ac.il, mp@ee.technion.ac.il). have been proposed, following approaches such as projection onto convex sets [5], stochastic and deterministic regularization [6], and iterative back-projection [7], among others. A different class of SR techniques is example-based $\mathrm{SR}$, in which correspondences between the low and high resolution images are learned from a database of low and high resolution image pairs [8]. Another approach that has recently been proposed makes use of a single image and is related to the Non-Local-Means (NLM) algorithm [9-10].

The majority of image interpolation and super-resolution algorithms focus on the interpolation of gray-scale images. In order to apply these algorithms to color images, an extension must be made in order to include all three color components. Separate interpolation of the channels has the potential of introducing color artifacts since RGB ratios might not be preserved. A common approach is to apply a sophisticated interpolation technique to the intensity component, and a simpler scheme (such as bi-cubic interpolation) to the color components. This is justified by the fact that the human eye is more sensitive to change of intensity than it is to color. While this approach achieves reasonable performance, some blurring still occurs at color edges due to the linear interpolation. In addition, correlation between the color and the intensity is not used. Several algorithms were designed specifically for color images [11].

In this work we present a SR algorithm that up-scales color textures using only information from the interpolated image. Textures are an important part of natural images, and their perception is believed to have a significant role in the process of recognition of the human visual system. Due to their importance, several approaches to texture analysis have been investigated over the years, such as statistical based techniques [12], techniques based on the Markov random field (MRF) model [13] and frequency domain techniques [14]. The MRF model has also been used for texture interpolation [15]. A different approach that has been used for texture filtering [16] and interpolation [10] is NLM. In this work we present a new approach for texture interpolation that is based on a single image. The proposed method has two steps. First, a stochastic interpolation of the intensity is performed based on local features, non-local patch similarity, as well as information from the color components. In the second stage, the color components are interpolated in a stochastic manner, using the already interpolated intensity values. The stochastic interpolation preserves the statistical relations between neighboring pixels and is shown to outperform existing methods.

The rest of the paper is organized as follows: the proposed algorithm is presented in Section II. In Section III interpolation results are presented. Section IV summarizes the proposed method and its performance. 


\section{The Proposed Algorithm}

Unlike [18], we are considering only same scale patch similarity. For each interpolated pixel, we compare the surrounding pixels, which we will refer to as the high resolution patch, to patches in the low resolution image.

In order to perform the comparison correctly, we take a preliminary scale adjustment step. In Fig. 1, an expansion of an image by a factor of two is shown (gray pixels are the available low resolution pixels). In this case, three types of pixels need to be interpolated - ' $h$ ', ' $v$ ' and 'd' type pixels.

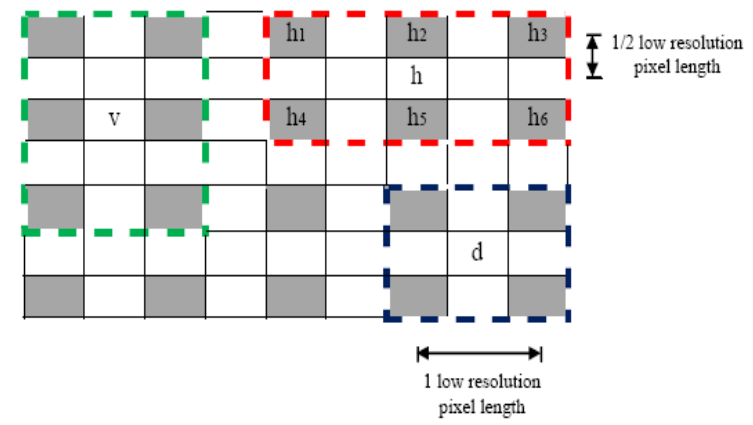

Fig. 1. Expanded image: gray- original (low resolution) pixels, whitemissing pixels

Considering for example the ' $\mathrm{h}$ ' type pixels, a corresponding low resolution patch used for the comparison is shown in Fig. 2.

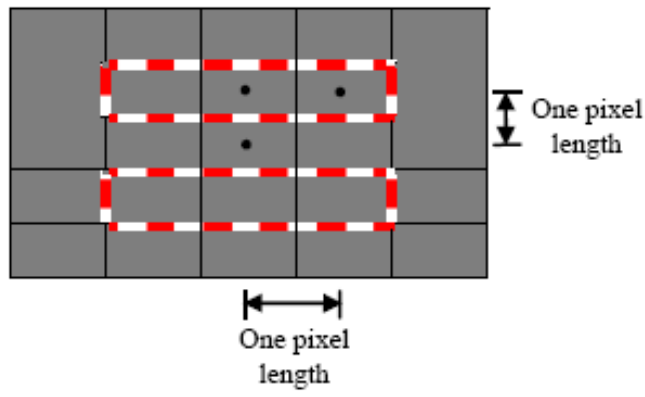

Fig. 2. Low resolution patch for ' $\mathrm{h}$ ' type pixels interpolation

Comparing the patch proportions in Figs. 1 and 2, a difference in the vertical direction can be seen. In order to use low and high resolution patches of equal proportions, we estimate the intermediate values by averaging every two rows as shown in Fig. 3.

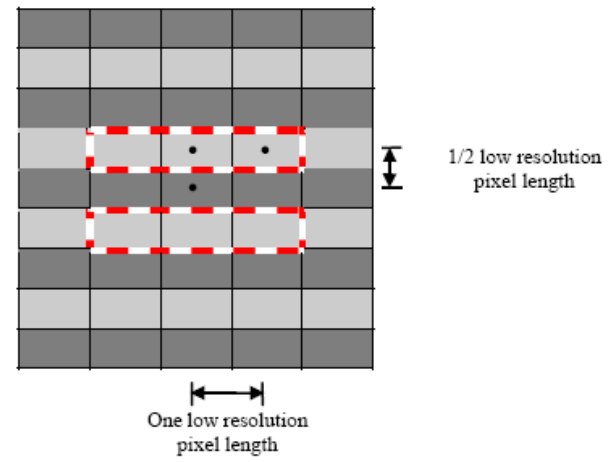

Fig. 3. Expansion of the low resolution image. dark gray rows - low resolution pixels, intermidiate rows - row averages

A similar procedure is used for the interpolation of ' $v$ ' type pixels. When comparing a high resolution patch to the low resolution patches, either the original or the modified low resolution image is used, as described in the following section.

\section{A. Interpolation of the Intensity Component Local - Non-Local Based Probability Distribution:}

Due to the repetitive nature of textures, several low resolution patches similar to the high resolution patch typically exist. The intensity values of the central pixels of these patches could be considered to be potential candidates for the intensity value of the interpolated pixel. In order for the interpolation process to preserve the statistical relations between neighboring pixels, we form a probability distribution for these values, according to which the interpolated value is chosen. The probability distribution takes into account local as well as non-local features of the texture. The non-local part ranks the candidate gray level values according to patch similarity regardless of the patch location, while the local part considers characteristics of the surrounding pixels such as smoothness. The probability distribution thus combines global statistics ('typical' intensity values for the particular pattern), with local features, which are also indicative of the gray-level probability distribution. The probability assigned to gray level $k$ is-

$$
P_{L-N L}(k)=\frac{w_{N L}(k) \cdot w_{L}(k)}{\sum_{i} w_{N L}(i) \cdot w_{L}(i)}
$$

where $w_{L}(k)$ and $w_{N L}(k)$ are the local and non-local weights that are assigned to each gray level $k$.

Non-Local Weights-

In [15], Buades et al. used a non-local filter (NLM) for the purpose of image denoising. We use a similar expression as the weight that is assigned to each low resolution patch reflecting its similarity to the high resolution patch.

When comparing a high resolution patch to low resolution patches, we consider two cases -

$$
\text { - } \operatorname{abs}\left(h_{2}-h_{5}\right) \leq d_{\text {thresh }}
$$

where $h_{2}$ and $h_{5}$ are the closest low resolution pixels (Fig. 1). In this case, the high resolution pixels $h_{1}, \ldots, h_{6}$ are compared to pixels from the averaged rows (Fig. 3 ).

$$
\text { - } \operatorname{abs}\left(h_{2}-h_{5}\right)>d_{\text {thresh }}
$$

The comparison is to low resolution image patches (Fig. 2).

The distinction is made since the row averaging causes smoothing of edges. The second condition is indicative of an edge, in which case the smoothed values are not used.

For a gray scale image $I(i, j)$ of size $N \times M$, the weight that is assigned to the low resolution patch centered at $(i, j)$ is -

$$
\begin{gathered}
=\exp \left\{-k_{1} \sqrt{S S D\left[Q\left(N_{\text {low }}(i, j)\right), Q\left(N_{\text {high }}(l, m)\right)\right]}\right\} \\
2 \leq i \leq N-1,2 \leq j \leq M-1
\end{gathered}
$$

where $N_{\text {high }}(l, m)$ is the group of pixels surrounding the interpolated pixel at $(l, m)$ in the high resolution image, $N_{\text {low }}(i, j)$ is the corresponding group of pixels of the low resolution patch centered at $(i, j)$, and $Q(\cdot)$ is a linear 
quantization function that is applied so that imperceptible differences have no effect on the assigned weights. The weight assigned to gray level $k$ is the following sum:

$$
w_{N L}(k)=\sum_{(i, j) \in I_{k}} \overline{w_{N L}}(i, j)
$$

where $I_{k}=\{(i, j) \mid I(i, j)=k\}$.

Local Weights-

The local weight $\mathrm{w}_{\mathrm{L}}(\mathrm{k})$ is calculated using a larger neighborhood, such as the one shown in Fig. 4 for ' $h$ ' type pixels.

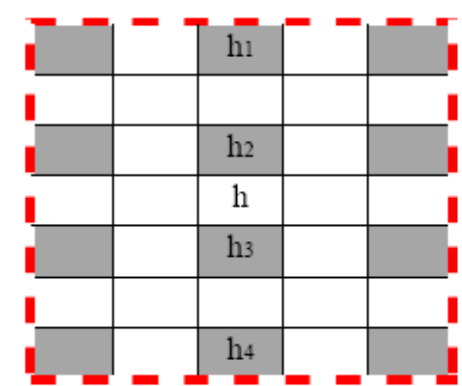

Fig. 4-Pixels used for the local weight calculation of 'h' type pixels

The local weight is a Gaussian whose mean and variance are determined by the neighboring low resolution pixel values (Fig. 4) -

$$
w_{L}(k)=\frac{1}{\sqrt{2 \pi \sigma_{L}^{2}}} \exp \left(-\frac{\left(k-\mu_{L}\right)^{2}}{2 \sigma_{L}^{2}}\right)
$$

Again, we consider two cases: edge and non-edge.

- $\operatorname{abs}\left(h_{2}-h_{3}\right) \leq d_{\text {thresh }}$

In this case $-\mu_{L}=\left(h_{2}-h_{3}\right) / 2, \sigma_{L}^{2}=\frac{1}{c} \operatorname{var}(N(l, m))$

- $\quad \operatorname{abs}\left(h_{2}-h_{3}\right)>d_{\text {thresh }}$

In this case the Gaussian mean was calculated using the inverse gradient approach, and the variance was proportional to the edge slope.

Using color-intensity correlation-

In many natural images the intensity and the color components are correlated. This correlation could be used in order to further improve the interpolation of the intensity component. In addition, a large class of textures (e.g., carpets, fabrics, etc.) contains only a small number of distinguishable colors, especially when several threads of different colors are used. For that reason, the color components, in the CIE-LA*B* space are first jointly quantized (using the k-means or a similar algorithm).

A threshold function is first applied to the probability distribution obtained from the intensity values in (1), setting small probabilities to zero.

$$
p_{L-N L}^{\prime}(k)=\left\{\begin{array}{cl}
P_{L-N L}(k) & P_{L-N L}(k)>P_{\text {thresh }} \\
0 & P_{L-N L}(k) \leq P_{\text {thresh }}
\end{array}\right.
$$

Next, weights are assigned to the gray level values based on the color components. The quantized color components of the high resolution patch are compared to those of the low resolution patches (in this case using Euclidian distances).
The more similar the color components are, the greater the weight that is given to the intensity value of the central pixel of the low resolution patch. As before, summation over all of the patches gives the weight of gray scale level $\mathrm{k}-\mathrm{w}_{\text {color }}(\mathrm{k})$. The probability distribution according to which the intensity value is chosen is

$$
p(k)=\frac{p_{L-N L}^{\prime}(k) w_{\text {color }}(k)}{\sum_{i} p_{L-N L}^{\prime}(i) w_{\text {color }}(i)}
$$

This completes the interpolation of the intensity for ' $\mathrm{h}$ ' and ' $v$ ' type pixels. The interpolation of ' $d$ ' type pixels proceeds in a similar manner, except that the already interpolated ' $h$ ' and ' $\mathrm{v}$ ' pixel values $\left(\mathrm{h}_{1}^{\prime}, \mathrm{h}_{2}^{\prime}, \mathrm{v}_{1}^{\prime}, \mathrm{v}_{2}^{\prime}\right.$ in Fig. 5) are used as well.

\begin{tabular}{|c|c|c|}
\hline$h 1$ & $v 1$ & $h 2$ \\
\hline$h 1$ & $\mathrm{~d}$ & $h^{\prime} 2$ \\
\hline$h 4$ & $v^{\prime} 2$ & $h 3$ \\
\hline
\end{tabular}

Fig. 5. Patch values used for interpolating 'd' type pixels

\section{B. Interpolation of the Color Components}

In the second stage of the algorithm, the color components are interpolated. A similar methodology as before is used, considering non-local similarity and local features. The color interpolation, however, relies on the previously interpolated intensity values. The color interpolation is based on a quantized version of the color components, i.e. quantized patches are compared. The color components, $\mathrm{A}^{*} \mathrm{~B}^{*}$, are associated with one of the values in the set- $C_{q}=$ $\left\{c_{1}, c_{2}, \ldots, c_{k}\right\}=\left\{\left(a_{1}^{*}, b_{1}^{*}\right),\left(a_{2}^{*}, b_{2}^{*}\right), \ldots,\left(a_{k}^{*}, b_{k}^{*}\right)\right\}$. In addition, the values that are assigned to the missing color components of the high resolution pixels are also elements of $\mathrm{C}_{\mathrm{q}}$.

The color that is assigned to each high resolution pixel is chosen, as before, according to a probability distribution, constructed from two weights, local and non-local, that are assigned to each value of $\mathrm{C}_{\mathrm{q}}$. The probability distribution is calculated from the weights according to (7).

$$
p\left(c_{r}\right)=\frac{w_{\text {color }-N L}\left(c_{r}\right) w_{\text {color }-L}\left(c_{r}\right)}{\sum_{i=0}^{k} w_{\text {color }-N L}\left(c_{i}\right) w_{\text {color }-L}\left(c_{i}\right)}, 1 \leq r \leq k
$$

As before, we first interpolate ' $h$ ' and ' $v$ ' type pixels.

Non-Local Weights-

Let us denote the group of pixels surrounding the pixel whose color is interpolated by $\mathrm{n}_{1}, \ldots, \mathrm{n}_{6}$. We denote their quantized color components by $c_{n 1}, \ldots, c_{n 6}$.

The high resolution patch of quantized color components is compared to corresponding patches of the quantized colors in the low resolution image. This time, however, only exact matches are considered. We form a set of the low resolution patches whose quantized color components are $c_{n 1}, \ldots, c_{n 6}$. We denote this group by $P=\left\{P_{1}, \ldots, P_{J}\right\}$, where $J$ is the number of such patches. Due to the typically small number of distinguishable colors in textures, and their repetitive nature, in most cases this group will be non-empty. In case the group is empty, the interpolated color is one of the two quantized colors of the nearest low resolution pixels. In this case the color is chosen using the color-intensity correlation. 
Let us now denote by $\left\{c_{p 1}, \ldots, c_{p L}\right\} \in C_{q}$ the quantized color components of the central pixels of the patches in $\mathrm{P}$, and by $\left\{i_{p 1}, \ldots, i_{p L}\right\}$ the intensity values of these pixels. In addition, for each quantized color $c_{r} \in C_{q}$ let us denote the group of indices corresponding to patches in group $\mathrm{P}$ for which the quantized color of the central pixel is $c_{r}$ by $J_{r}=\left\{j \mid c_{j}=c_{r}\right\}$. The non-local weight is then defined by

$$
w_{\text {color }-N L}\left(c_{r}\right)=\sum_{j \in J_{r}} \exp \left(-k_{2} \cdot\left(i_{p j}-i^{*}\right)^{2}\right)
$$

where $i^{*}$ is the interpolated intensity value of the currently interpolated pixel. It can be seen from (8), that in order for a color in $C_{q}$ to have a non-zero probability, it must be the quantized color of a central pixel in one of the patches.

\section{Local Weights-}

The purpose of local weights is to ensure that the interpolation process does not introduce any colors that are different than the colors of the surrounding pixels. Let us denote the (quantized) color components of the high resolution patch by $\left\{\mathrm{c}_{1}, \ldots, \mathrm{c}_{6}\right\}$. The weight assigned to each possible color is

$$
w_{\text {color-L }}\left(c_{i}\right)= \begin{cases}1 & c_{i} \in\left\{\mathrm{c}_{1}, \ldots, \mathrm{c}_{6}\right\} \\ 0 & c_{i} \notin\left\{\mathrm{c}_{1}, \ldots, \mathrm{c}_{6}\right\}\end{cases}
$$

The interpolation of the ' $\mathrm{d}$ ' type pixels proceeds in the exact same manner, except that the color components that have already been interpolated are used as well.

\section{EXPERIMENTAL RESULTS}

\section{A. Performance Evaluation of the Local-Non-local approach}

In order to evaluate the interpolation of the intensity, a $100 \times 100$ portion of the (gray scale) wood texture from the Brodatz database [17] was interpolated. The full (high resolution) texture was first downscaled by a factor of 2 , and then up-sampled. The interpolation using the proposed algorithm relied on information from a larger portion of the image (256x256 pixels).
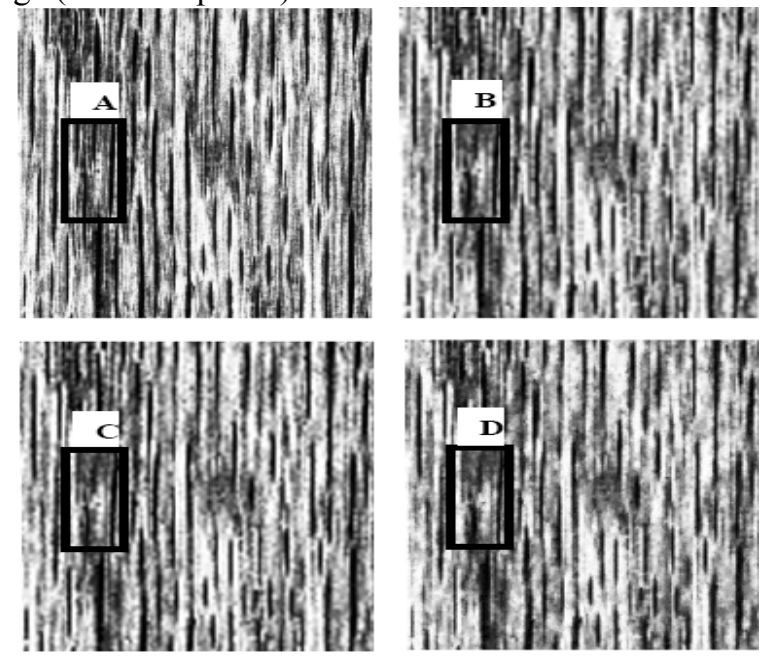

Fig. 6. Texture 'wood' . top left - high resolution image, top right - bicubic interpolation, bottom left - cubic spline interpolation, bottom right - the proposed method
The PSNR values of the interpolated "Wood" texture are presented in the following table:

\begin{tabular}{|l|c|}
\hline & PSNR(dB) \\
\hline Bicubic interpolation & 14.1 \\
\hline Cubic splines interpolation & 15.8 \\
\hline The proposed algorithm & 15.8 \\
\hline
\end{tabular}

As can be seen, the PSNR value that was obtained using the proposed interpolation method is the same as that obtained using cubic splines. In Figs. 6 and 7 the interpolated "Wood" textures are presented. It can clearly be seen that the interpolation using the proposed method produces a significantly less blurry texture in comparison to the other interpolation methods. The proposed interpolation algorithm was also applied to other textures from the Brodatz database, yielding similar results, i.e. PSNR values similar to those obtained using cubic splines and superior to bicubic interpolation, while being less blurry than both.
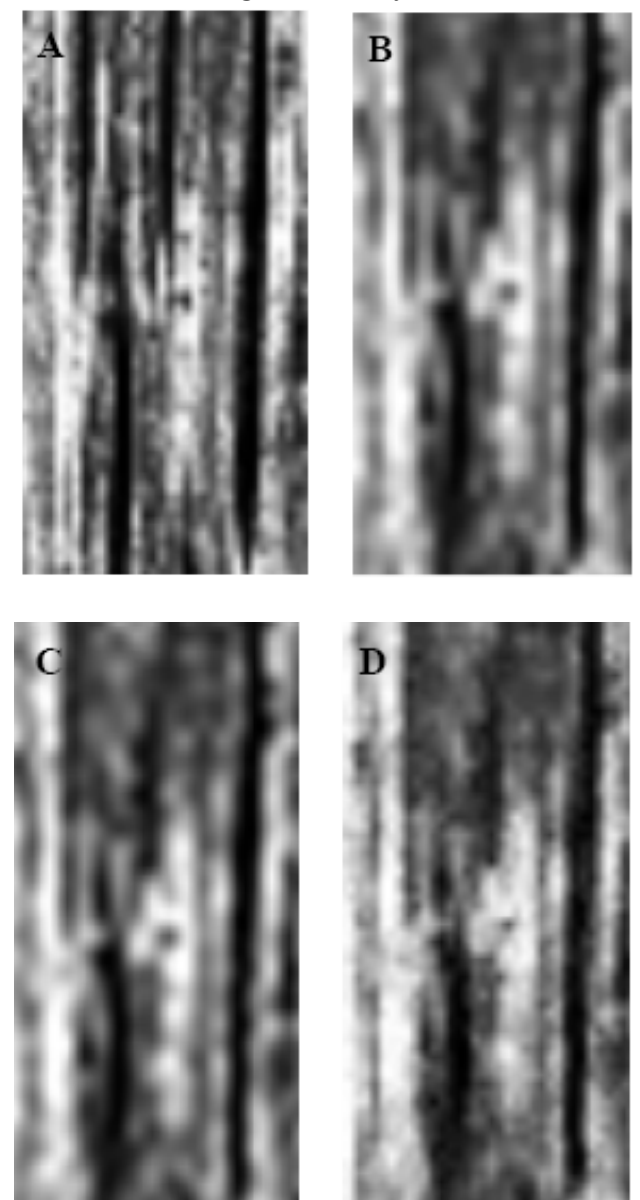

Fig. 7. Magnified patches as marked in Fig. 6.

\section{SUMMARY AND CONCLUSIONS}

In this paper we have introduced a single-image super-resolution method that is able to successfully interpolate a very broad class of textures - stationary as well as non-stationary. Unlike other interpolation techniques, the proposed method incorporates the information in the color channels in the interpolation of the intensity values. As indicated by the results, using information from local characteristics as well as from similar patches achieves high 
resolution images that are visually superior to presently available interpolation techniques.

\section{REFERENCES}

[1] X. Li and M. T. Orchard, "New edge-directed interpolation," IEEE Trans. Image Process, vol. 10, no. 10, pp. 1571-1527, Oct. 2001.

[2] A. Belahmidi and F. Guichard, "A partial differential equation approach to image zoom," In Proc. of the Int. Conf. on Image Processing, pp. 649-652, 2004.

[3] S. Esedoglu and J. Shen, "Digital inpainting based on the Mumford-Shah-Euler image model," European Journal of Applied Mathematics, Vol. 13, pp. 353-370, 2002.

[4] S. Park, M. Park, and M. Kang, "Super-resolution image reconstruction: a technical overview," IEEE Signal Process. Mag, vol. 20, no. 3, pp. 21-36, May 2003.

[5] H. Stark and P. Oskoui, "High resolution image recovery from image-plane arrays, using convex projections," J. Opt. Soc. Am. A, vol. 6, pp. $1715-1726,1989$.

[6] R. C. Hardie, K. J. Barnard, and E. E. Armstrong, "Joint MAP registration and high-resolution image estimation using a sequence of undersampled images," IEEE Trans. Image Processing, vol. 6, pp. 1621-1633, Dec. 1997.

[7] M. Irani and S. Peleg, "Improving resolution by image registration," CVGIP: Graphical Models and Image Proc, vol. 53, pp. 231-239, May 1991.
[8] K. Kim and Y. Kwon. "Example-based learning for single image SR and JPEG artifact removal," Technical Report 173. Max Plank Institute, August 2008.

[9] D. Glasner, S. Bagon, and M. Irani, "Super-resolution from a single image," in International Conference on Computer Vision, 2009.

[10] T. Wittman. "Mathematical Techniques for Image Interpolation," Report Submitted for Completion of Mathematics Department Oral Exam, Department of Mathematics, University of Minnesota, USA, 2005.

[11] Y. W. Tai, W. S. Tong, and C. K. Tang, "Perceptually-inspired and edge-directed color image super-resolution," In $C V P R$, vol. 2, pp. 1948-1955, 2006

[12] J. Weszka, C. Deya, and A. Rosenfeld, "A Comparative Study of Texture Measures for Terrain Classification," IEEE Trans. System, Man and Cybernetics, vol. 6, pp. 269-285, 1976.

[13] H. Yin and N. Allinson, "Unsupervised Segmentation of textured Images Using a Hiererchical Neural Structure," Electronics Letters, vol. 30, no. 22, pp. 1842-1843, 1994.

[14] M. Porat and Y. Zeevi, "Localized Texture Processing in Vision Analysis and Synthesis in the Gaborian Space," IEEE Trans. Biomedical Eng, vol. 36, pp. 115-129, 1989.

[15] S. Nemirovsky and M. Porat, "On Texture and Image Interpolation using Markov Models," Signal Processing and Image Communication, vol. 24, pp. 139-157, 2008.

[16] A. Buades, B. Coll, and J.M. Morel, "On image denoising methods, "CMLA Preprint, CMLA pp. 2004-2015, 2004.

[17] P. Brodatz. Dover, New York, 1966. 